and trigger PSA (OR 1.022; $P<0.001$ ) to be associated with a positive BS. A nomogram was subsequently constructed to predict a positive result.

Dotan et al. conclude that the nomogram represents an important, user-friendly tool allowing the physician to identify high-risk and low-risk patients for systemic progression, according to preoperative and postoperative variables and pattern of PSA failure any time during the patient's followup. The use of multiple predictors makes the approach more accurate, as using trigger PSA alone is not a reliable predictor. A randomized trial is needed to evaluate whether early treatment for high-risk patients will lead to improved survival and a reduction in metastatic rate.

Original article Dotan ZA et al. (2005) Pattern of prostatespecific antigen (PSA) failure dictates the probability of a positive bone scan in patients with an increasing PSA after radical prostatectomy. J Clin Oncol 23: 1962-1968

\section{4-hour urinary calcium as a marker for dietary calcium intake}

The negative correlation between dietary calcium intake and risk of recurrent calcium-based stones formed the basis of a recent study that investigated whether $24 \mathrm{~h}$ urinary excretion of calcium could form a reliable marker for dietary intake of calcium.

Toren and Norman randomly selected 68 women from their stone clinic. All had attended the clinic at least twice and had been recruited to complete a four-day record of their diet along with two $24 \mathrm{~h}$ urine collections on days three and four. The researchers analyzed the dietary data and composition of urine samples provided by participants, and used multiple regression analysis to investigate the relationship between individual dietary nutrients and the mean $24 \mathrm{~h}$ urinary calcium level.

A low $24 \mathrm{~h}$ urinary calcium level did not always correlate with a low dietary intake of calcium. Furthermore, statistical analysis suggested that the positive predictive value of a patient's $24 \mathrm{~h}$ urine calcium level was not sufficient to be considered a reliable surrogate marker for their respective dietary calcium intake.
This study reinforces the need to obtain detailed dietary records for patients with stone formation so that their calcium intake can be modified where necessary, to minimize the risk of stone recurrence.

Original article Toren PJ and Norman RW (2005) Is 24hour urinary calcium a surrogate marker for dietary calcium intake? Urology 65: 459-462

\section{Do patient preferences reduce the validity of randomized trials?}

Although a randomized controlled trial is generally accepted as the most effective way to assess clinical efficacy, patients' preferences for a particular treatment might affect the study's validity. This is particularly relevant when treatments are not blinded, and is likely to become increasingly important as patients take an ever more active interest in their management.

To assess the magnitude of any effect of patient choice on recruitment or outcomes, King and colleagues have carried out a systematic review of clinical studies that recorded patient or physician treatment preference. The selected studies followed up all participants, whether allocated to random or preference cohorts.

The results revealed that a considerable proportion of patients refused randomization because they preferred one treatment over another. In 14 of 27 studies included in the analysis, more than $50 \%$ of individuals refused randomization after having agreed to participate in the trial. Reassuringly, however, there was little bias in the characteristics of those who were randomized, and only small differences were found between the randomized and preference groups in terms of outcomes.

King et al. conclude that although recruitment to randomized trials is strongly affected by patients' preferences, neither external nor internal validity appears to suffer significantly. These findings also lend support to the use of observational studies in areas where randomization is not appropriate.

Original article King M et al. (2005) Impact of participant and physician intervention preferences on randomized trials: a systematic review. JAMA 293: 1089-1099 